\title{
A study of embolism in mitral valve disease and atrial fibrillation
}

\author{
H A DEWAR, D WEIGHTMAN \\ From the Royal Victoria Infirmary and University Department of Medical Statistics, Newcastle upon Tyme
}

SUMMARY A number of possible risk factors, clinical, radiological, biochemical, and social have been examined in one group of 34 cases of mitral valve disease and four of lone atrial fibrillation, all of whom gave a history of embolism, and also in a group of 24 cases of mitral valve disease who gave no such history. All were on long-term anticoagulant treatment. Comparison of the two groups disclosed no features that would distinguish those who ran the greater risk of embolism from the remainder other than that the men in the former group were older and of shorter stature. At comparable age, the women with a history of embolism had smaller left atria. Examination of a special group of nine patients who had had embolism despite anticoagulant treatment also disclosed no special features, other than a greater age at the time of their first embolism. Fibrinolytic activity was less and the level of beta thromboglobulin was greater than normal in both groups. Those with proven risk of embolism were not distinguished by higher values of this platelet protein. Neither variable correlated with the type of heart disease. No positive association was found between cigarette smoking and the risk of embolism. Embolism was significantly more likely to occur between 0801 and 1600 hours than at other times of day.

It has been known for several decades ${ }^{1}$ that while mitral valve disease or atrial fibrillation alone may cause embolism the combination of the two is particularly dangerous. In most clinics therefore patients with this combination are treated with anticoagulants of the coumarin type, with good, though not invariably successful, protective effect. ${ }^{2-4}$

Yet there are some puzzling features which have been pointed out in most of the large-scale studies already published.4-6 Thus, embolism may occur in cases of mitral stenosis still in sinus rhythm $(20 \%$ in three series ${ }^{4}{ }^{7}$ ) or when it is mild, or when there is pure regurgitation, and even when with anticoagulant treatment the effect is within the therapeutic range. The risk also bears no definite relation to size of heart as a whole or to that of the left atrium. On the other hand, other patients with mitral disease and atrial fibrillation or the latter alone will pass many years unprotected by any treatment and still have no emboli. In clinical practice therefore some patients seem to be receiving treatment that carries its own risks and that they do not need, while others are

Accepted for publication 11 November 1982 receiving "good" treatment that is nevertheless a failure.

It was decided therefore to study some of the other factors that might be relevant and to see if markers could be found that would identify those patients who needed preventive treatment and to define what form it should take. Even when coumarin-type anticoagulants were introduced many years ago, it was already known that the potential emboli which formed in the left atrium had the structure of arterial platelet thrombi rather than of fibrinous venous clots, and on the whole the treatment has been surprisingly successful. In recent years, however, the part played by platelets in the formation of these thrombi has been studied, Steele et al. ${ }^{8}$ having shown reduced platelet survival and Toy et al. ${ }^{9}$ a correlating increase of platelet stickiness. In fact, the respective parts played by fibrin deposition and by platelet adhesiveness and aggregation in cases of mitral valve disease must vary from case to case, for Rozenberg and Dintenfass ${ }^{10}$ have shown experimentally that the structure of thrombi and the relative proportions of fibrin and platelets in them is largely determined by rates of blood flow. Where it is high, they have the typical structure of arterial thrombi, with large masses of 
aggregated platelets in a rather scanty network of fibrin. Where, however, rate of flow is low, they have the structure of venous clots, with individual or small conglomerates of platelets widely scattered in numerous strands of fibrin. One might expect therefore that in a case of mitral stenosis that was mild, where the left atrium was small, rhythm regular, and cardiac output only modestly reduced, any thrombus in that atrium would have a large platelet element, and that the blood might contain relatively large quantities of the products of their disintegration. Where, on the other hand, the left atrium was big, its wall fibrillating, and cardiac output very low, fibrin deposition would be greater. In these cases the capacity of the blood to lyse this fibrin might be of much importance.

In 1975 beta thromboglobulin was introduced as an extremely sensitive indicator of platelet destruction. Since its measurement involved the taking of only one or two samples of venous blood, it had very obvious advantages over studies of platelet survival time. Accordingly, in this investigation it was decided to evaluate beta thromboglobulin as a possible marker of those patients who needed prophylactic treatment against embolism and to see if its level in the blood would correlate with those types of mitral valve disease in which platelet turnover would be highest. Since the adhesive and aggregating properties of platelets are also responsive to factors such as cigarette smoking, the use of the contraceptive pill, and bodily activity and posture, it was decided to investigate these too in relation to risk and occurrence of embolism. Blood values of fibrinogen, cholesterol, and triglycerides (fasting) would also be estimated.

With regard to the tendency to deposition of fibrin, it was decided not to measure degradation products, because any substantial number of patients recruited into the study would be bound already to be receiving anticoagulant treatment. The fibrinolytic activity of venous blood, which is little affected by such treatment, ${ }^{11}$ would appear to have more relevance, and the euglobulin lysis time in antecubital vein blood would therefore be estimated on two occasions in the fasting and resting state and once when the patients were unrested and non-fasting. The lower of the former two estimations would be used in evaluation as reflecting the state of greater risk of thrombus formation. The 20 minute venous occlusion test, as standardised by Nilsson and Pandolfi, 12 was deliberately omitted, partly because of its doubtful clinical relevance, and partly because of the discomfort it causes patients. The state of fibrinolytic activity of antecubital vein blood is not, of course, the same as that in the left atrium, but previous cardiac catheterisation measurements ${ }^{13}$ have shown that though pulmonary arteries and veins both liberate activator into the circulation, the former much more so than the latter, fibrinolytic activator is almost certainly destroyed or. neutralised in the pulmonary capillaries. Euglobulin $\overrightarrow{\overrightarrow{\mid}}$ lysis time values in pulmonary veins, left atrium aorta, and peripheral arteries are identical, but substantially less than those in peripheral and centrate veins, right ventricle, and pulmonary artery, a feature that possibly explains why in clinical experience of patients with atrial fibrillation pulmonary emboli ares less frequent than systemic emboli. If levels were $\overrightarrow{0}$ found to be unusually low in antecubital vein blood, they would almost certainly be lower still in the lefiw atrium. It was proposed therefore not only to see how the antecubital vein value compared with normal, but? also whether that value correlated, especially $\overrightarrow{0}$ inversely, with the type of valve defect and clinical status in which intracardiac thrombi might be्w expected in theory at least to have a relatively higho fibrin content.

Previous studies ${ }^{14} 15$ had failed to show any $\vec{T}$ significant difference between the euglobulin lysiso time values of smokers and non-smokers but, in viewe of its importance, the possibility of such a difference would be tested again, and so far as possible the gen eral relevance of cigarette smoking to the risk of embolism would be assessed. The similar possible relevance of the use of contraceptive pills would alsoo be inquired into.

Finally, since in many instances patients witho mitral valve disease and a history of embolism haveू had valve operations or have died and neither the surgeon nor the pathologist, respectively, have foun $F^{+}$ thrombus in the left atrium, ${ }^{1617}$ it has been though likely that the thrombus has embolised as soon as it formed. It seemed interesting therefore to inquire into the exact circumstances of the patients to be studied in terms of posture, physical activity, and time of day when their embolism occurred.

For all these purposes therefore two groups o\$ patients, all of whom had been under the care of ones of us (HAD) and all of whom were on long-term anticoagulants, were recruited. Group 1 had sustained ones or more emboli, group 2 had not. Group 1 was further subdivided into those who have had no furthero emboli since beginning anticoagulants (group 1A). and those who had had further emboli despite sucho treatment well controlled (group 1B). Groups 2, 1A and $\mathrm{BB}$ therefore represented patients at progressive risk.

Later, when on analysis it appeared that one sex linked group differed significantly in respect of one of the circumstances in which emboli took place but the numbers were small, a further 10 patients were recruited from the clinics of colleagues and added to group 1. These were examined, however, solely ind relation to the circumstances attending their embol isms and to their smoking habits at the time. 


\section{Subjects and methods}

Group 1 comprised 38 patients, 11 men and 27 women, all of whom gave a history of embolism, 58 episodes in all, of which $45(78 \%)$ were cerebral. All except five were in atrial fibrillation at the time of this study but nine had sustained a total of 11 emboli while still in sinus rhythm. Sixteen of the $38(42 \%)$ had experienced an embolus within one year of the onset of atrial fibrillation. Four (two men, two women) had atrial fibrillation without valve disease, and the remainder all had rheumatic mitral valve disease, mostly stenosis. Five had had mitral valve replacements, but the emboli recorded had all preceded this operation; in these cases however beta thromboglobulin estimations have been omitted. Subgroup 1B comprised nine patients (two men, seven women) who had sustained a total of 13 further emboli while on anticoagulants. In eight of these the thrombotest was within the therapeutic range at the time of at least one of the emboli, and in one it was outside it. All these cases were in atrial fibrillation and their emboli were systemic. In order to make the three groups of progressive risk (groups 2, 1A, and 1B) comparable, we deducted from group 1A those patients who were not in atrial fibrillation, whose emboli were pulmonary, or who had further emboli despite anticoagulants, but whose state of control was unknown or poor at the time. The group 1A, therefore, who had been "protected" by treatment, consists of eight men and 14 women.

Group 2 comprised 24 patients, seven men and 17 women, all in chronic atrial fibrillation at the time of study, except two in whom the arrhythmia was intermittent. All had rheumatic mitral valve disease, mostly stenosis. None had suffered embolism and none had valve replacements.

The extra 10 patients "lent" by colleagues for study of the circumstances of their emboli and of their smoking habits were all men, had mitral valve disease, gave a history of embolism, and had been on anticoagulants since. None had valve replacements at the time of their emboli.

The biochemical investigations in groups 1 and 2 were all made at the time this study was begun, in 1978-9. Their cardiac status, however-severity of valve defect, total heart size, and left atrial size-has been compared both at the time of this study and also at the period of greatest risk of embolism, ${ }^{3}$ that is during the first year after onset of atrial fibrillation. It was possible during that first year to compare 23 cases without embolism with 31 cases with embolism. The latter had been started on anticoagulants at once, but in the case of the ones without embolism such treatment had been delayed for at least a year in 12 of them and for at least six months in 14 , that is at least
$50 \%$ of them had been unprotected for the best part of a year. Severity of mitral obstruction and/or regurgitation was assessed from the clinical features, supported where available by $x$-ray, cardiac catheterisation, or operative findings and graded 0 to 4 . Heart size (cardiothoracic ratio) was measured where $x$-ray films were still available but accepted from reports where they were not. The figures for size of the left atrium were only recorded when $x$-ray films were available, enabling one to measure its transverse diameter from the right outermost border to the junction of the lower border of the appendage and the body. This was then related to the transverse diameter of the thorax at the same level. The history of cigarette smoking, use of the contraceptive pill, and the circumstances of the episodes of embolism (which they remembered years after with remarkable clarity) were all obtained by up to date interview, rarely by correspondence.

Beta thromboglobulin was estimated by the standard radioimmunoassay method of Bolton et al. ${ }^{18}$ and fibrinolytic activity by the euglobulin lysis time method of Von Kaulla ${ }^{19}$ as modified by Menon et $a l .,{ }^{20}$ the reciprocal of the time in minutes being multiplied by 10000 to give units. Fibrinogen, cholesterol, and triglycerides were estimated by routine laboratory methods.

\section{STATISTICAL METHODS}

Student's $t$ test for independent samples, and Student's paired $t$ test for dependent samples were used. Fisher's exact probabilities test, exact confidence limits, and correlation coefficient were calculated, and the analysis of variance for the three groups of progressive risk was carried out.

\section{Results}

\section{CLINICAL FEATURES}

Tables 1 to 3 show the clinical features of groups 1 and 2 at the time of the study together with the beta thromboglobulin and euglobulin lysis time values. The men with emboli were older and shorter in stature than the men without emboli. As is usual with mitral valve disease, women considerably outnumbered men $(2.5: 1)$. Neither when the sexes were examined separately nor when examined together did those with a history of embolism differ from those without in respect of the severity or nature of their disease, in the size of their hearts, or of their left atria, in the years of observation, or in the years of atrial fibrillation. An analysis (not shown) of the size of the left atria at the time when they first came under observation showed that in the women who had not had embolism that chamber was significantly $(p<0.001)$ larger than it was in those who had suffered such episodes. The two groups of women were of very 
Table 1

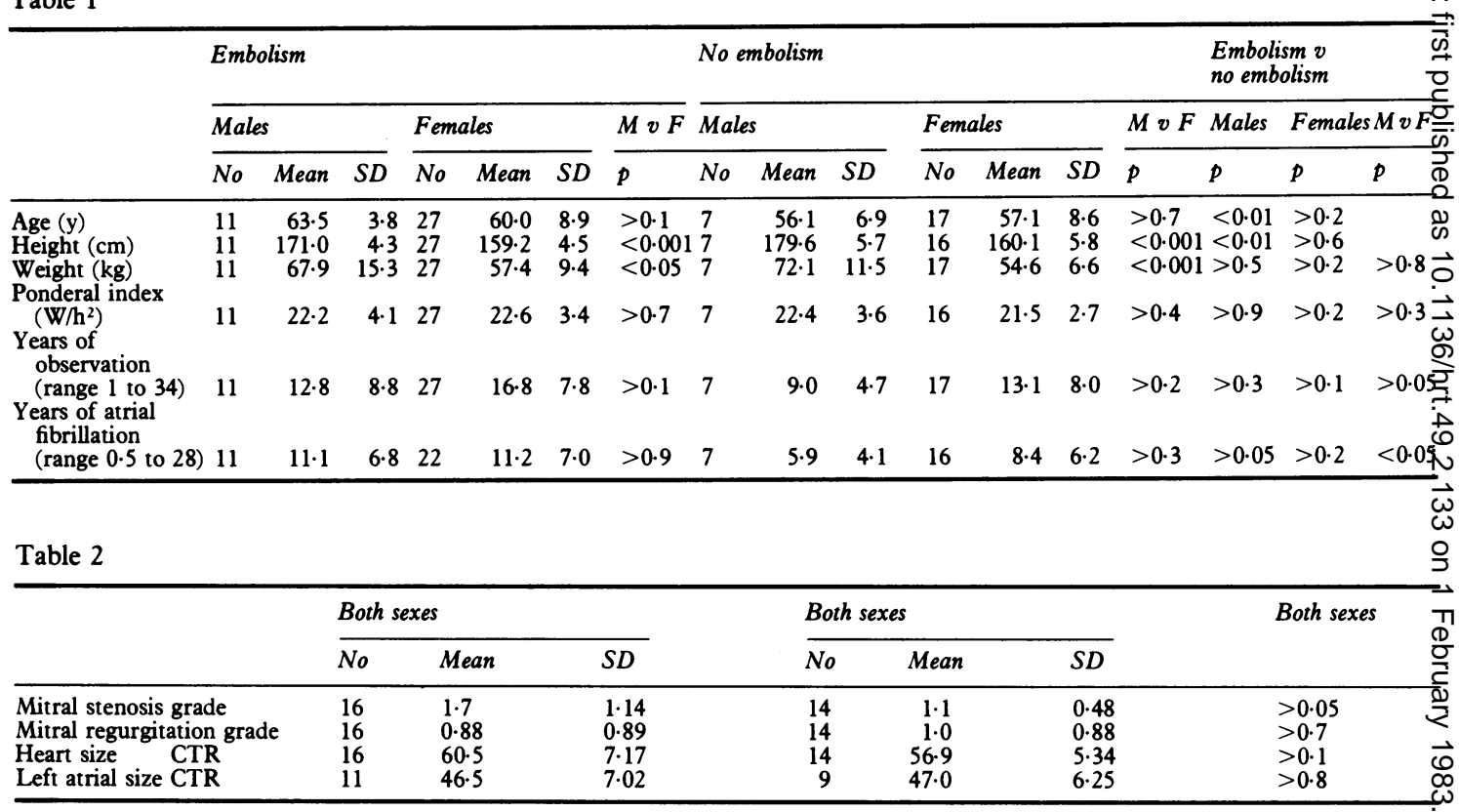

Table 3

\begin{tabular}{|c|c|c|c|c|c|c|c|c|c|c|c|c|c|c|c|c|c|}
\hline & \multicolumn{3}{|c|}{ Males } & \multicolumn{3}{|c|}{ Females } & \multirow{2}{*}{$\begin{array}{l}M v F \\
p\end{array}$} & \multicolumn{3}{|c|}{ Males } & \multicolumn{3}{|c|}{ Females } & \multicolumn{4}{|c|}{$M v F$ Males Females $M v F$} \\
\hline & No & Mean & $S D$ & No & Mean & $S D$ & & No & Mean & $S D$ & No & Mean & $S D$ & $p$ & $p$ & $p$ & $p$ \\
\hline Fibri & 9 & $41 \cdot 2$ & $34 \cdot 6$ & 24 & 33.8 & 37.8 & $>0.6$ & 7 & 39.4 & 18.1 & 17 & $31 \cdot 2$ & 23.4 & $>0.4$ & $>0.9$ & $>0.8$ & $>0.7$ \\
\hline $\begin{array}{l}\text { Activity - units } \\
\text { non-fasting } \\
\text { Beta thrombo- }\end{array}$ & 9 & $51 \cdot 0$ & $36 \cdot 2$ & 20 & $31 \cdot 2$ & 18.8 & $>0.1$ & 5 & 44.4 & $35 \cdot 1$ & 14 & 258 & 18.4 & $>0.4$ & $>0.7$ & $>0.4$ & $>0.9$ \\
\hline globulin & 8 & 1.79 & 0.204 & 422 & 1.82 & 0.211 & $1>0.7$ & 5 & 1.88 & 0.151 & 14 & 1.883 & $0 \cdot 128$ & $>0.9$ & $>0.4$ & $>0.2$ & $>0.1$ \\
\hline Logs & & 61.4 & & & $65 \cdot 3$ & & & & $75 \cdot 2$ & & & 76.4 & & & & & \\
\hline
\end{tabular}

similar age (46.5 and 47.2 years, respectively) at the time. Valid comparison of this chamber at the time of onset of atrial fibrillation when risk of embolism is greatest was unfortunately not possible. Groups 2, $1 \mathrm{~A}$, and $1 \mathrm{~B}$ were of very similar mean age $(49.7,50.4$, and 50.7 years) when this arrhythmia appeared.

\section{BIOCHEMICAL INVESTIGATIONS}

Regarding fibrinolytic activity, expressed as units, the values in the group without embolism were 39 and 31 for men and women, respectively, in the group with embolism 41 and 34, and in subgroups $1 \mathrm{~A}$ and $1 \mathrm{~B}, 32$ and 42 (sexes combined). None of these differences is statistically significant. Nor are the changes in the non-fasting, unrested state to $\mathbf{4 4}$ and 36 in the group with no embolism, and to 51 and 31 in the groups with embolism. Attempts to find correlations with the size of the heart, or of its left atrium, or with large heart and low output states were also unsuccessful. 0

The levels of beta thromboglobulin did not differ significantly between the two groups nor between the subgroups, nor between the sexes. Because the meañ values (64.3 in group 1 and 76.1 in group 2) were high, a further analysis was made of the values in patients at or above 60 years of age and there proved to be no significant increase with advanced years.

The analyses described above were repeated on the three groups of progressive risk previously men tioned, namely group 2, and groups $1 \mathrm{~A}$ and $1 \mathrm{~B}$. The last had just significantly $(\mathbf{p}<0.05)$ smaller hearts and less mitral regurgitation and were also older than the rest, $60: 47$ years $(p<0.02)$ when they had their firs embolism, but did not differ in any other way.

Similar negative results were obtained in respect of 
Table 4 Smoking habits

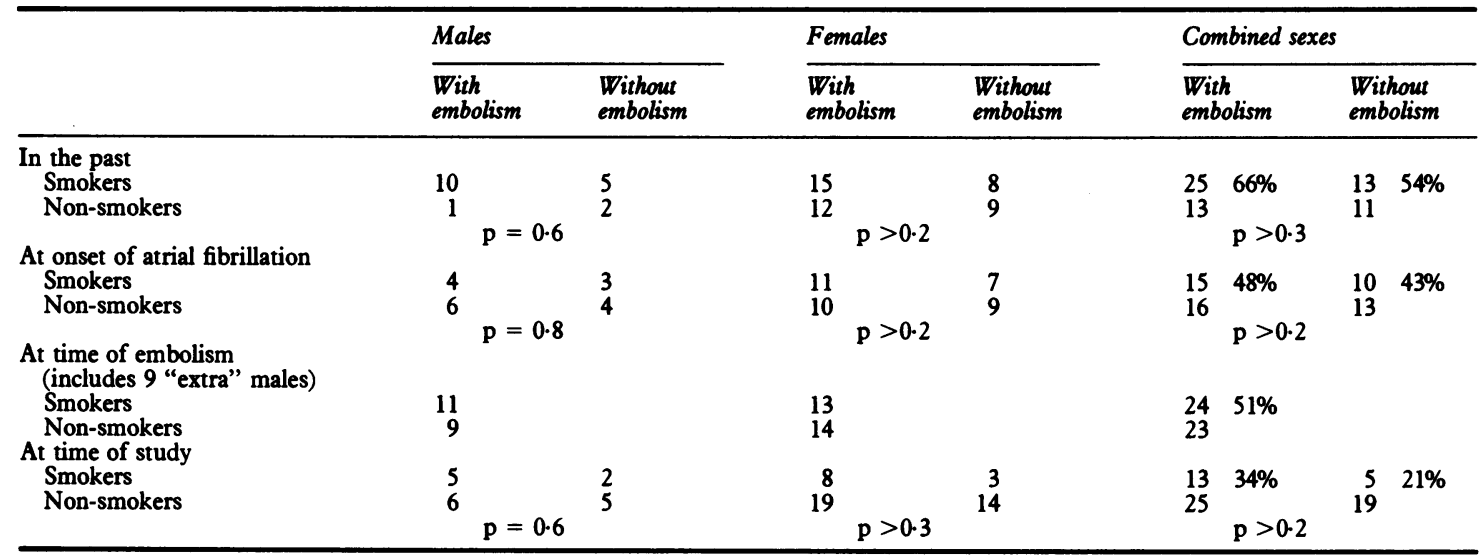

From the past to the present there is a significant decline in smoking in both the embolism $(p=0.001)$ and no embolism groups $(p=0.002)$. Of those who continued to smoke in the two groups only three did not reduce the amount smoked.

Table 5 Circumstances attending episodes of systemic embolism

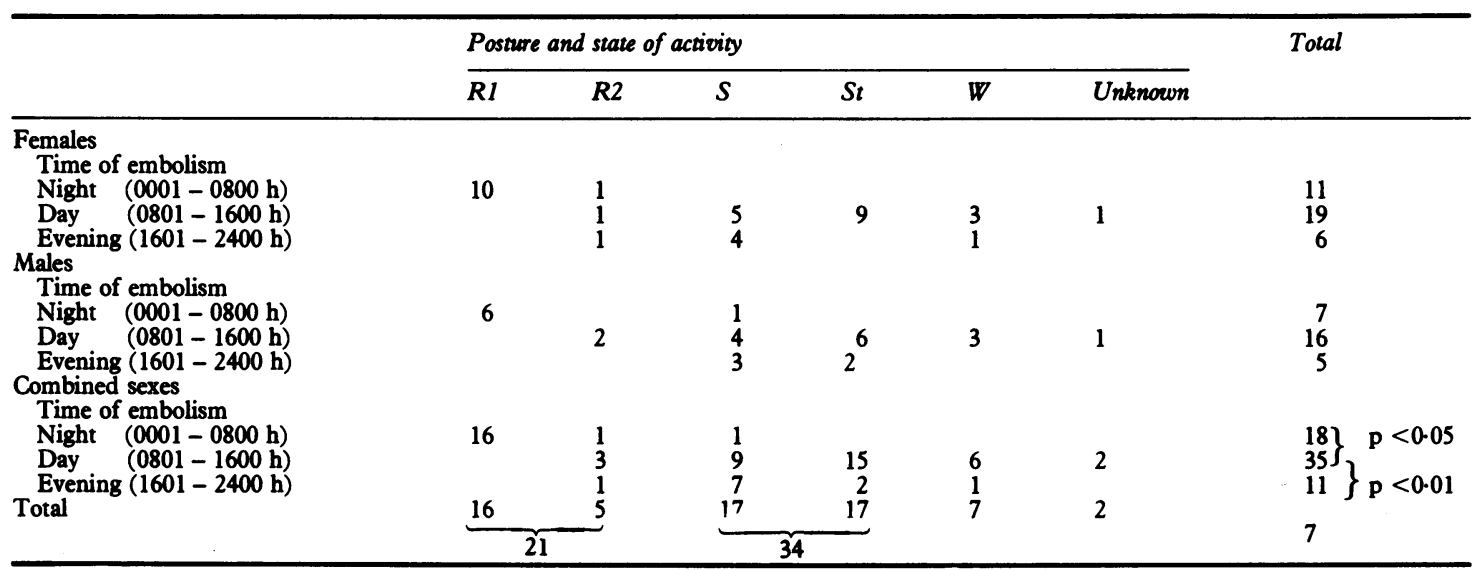

$R 1$, recumbent, asleep; R2, recumbent, awake; S, sitting; St, standing; $W$, walking (or more active still).

plasma fibrinogen, cholesterol, and triglycerides both in a comprison of the main groups 1 and 2 , and of the three subgroups of progressive risk.

\section{SMOKING HABITS}

Table 4 shows the smoking habits of groups 1 and 2 under three headings, namely in the past, at the time of onset of atrial fibrillation, and, most recently. at the time of this study. In addition, the smoking habits at the time of embolism of all those in group 1 are shown and to these has been added similar information which was available from nine out of the 10 extra men recruited to the group. Analysis, not shown in Table 4 , has also been made both of numbers of smokers and of the numbers of cigarettes smoked in the three groups of progressive risk, groups 2, 1A, and 1B, the first at the time of onset of atrial fibrillation, and the latter two similarly and also at the time of embolism. None of these comparisons yielded any statistical proof of the dangers of smoking in relation to the risk of embolism. The beta thromboglobulin values of current smokers were not significantly higher than those of current non-smokers. Both major groups, however, did show a highly significant reduction in the prevalence of the smoking habit over the years of observation.

\section{CONTRACEPTIVE PILL}

So far as the possible risk attached to use of a contraceptive pill is concerned, only one patient was taking such a pill at the time of her embolus, and only two in the non-embolic group were doing so during the relevant periods of observation. 
CIRCUMSTANCES OF EMBOLISM

Table 5 shows the distribution of emboli in three eight-hour periods of the 24, and the pattern of the sexes is the same. When the sexes are combined the day period, 0801 to 1600 hours, can be seen to include a significantly larger proportion of the emboli than the night period 0001 to 0800 hours $(p<0.05)$ or the evening period 1601 to 2400 hours $(p<0.01)$. A significantly $(p<0.05)$ small proportion of the emboli occurred when the patient was recumbent. In the "special risk" subgroup $1 \mathrm{~B}$, nine out of 15 emboli also occurred during the period 0801 to 1600 hours.

\section{OTHER COMPARISONS AND CORRELATIONS}

When in the embolism group, patients with hearts favouring platelet thrombi in the left atrium have been compared with hearts favouring more fibrinous thrombi there, as described in the introduction (both comparisons being made at the time of first embolism), no significant differences were detected in their level of beta thromboglobulin or of fasting or nonfasting euglobulin lysis times.

In addition, a number of other comparisons between groups 1 and 2, and between groups 2, 1A, and $1 \mathrm{~B}$ have been made and correlations tested, all with negative results. The comparisons included cardiac index and other variables measured at cardiac catheterisation. The correlations included years of atrial fibrillation with fasting lysis times, and beta thromboglobulin with cardiac index, heart and left atrial size, and lysis times.

\section{Discussion}

The predominantly negative results of most of the clinical comparisons are not surprising. They fit in well with previous experience ${ }^{4}$ that while risk of embolism is closely related to the presence of mitral valve disease and much increased by the presence of atrial fibrillation, it is not related to the degree of obstruction however measured, though Casella et al. ${ }^{5}$ did indeed find a significantly lower cardiac index in patients who had had emboli. Fleming and Bailey ${ }^{4}$ made a particular study of the size of the left atrial appendix in their comparison of mitral valve cases with and without embolism, since Somerville and Chambers $^{21}$ had suggested a positive relation. They found none. In the present study, the left atria as a whole were graded, and in women the left atria of the embolic group studied at comparable mean age were significantly smaller. Likewise, subgroup $1 B$, who had repeated emboli despite anticoagulants, was not distinguished by the largeness of their hearts as a whole nor of their left atrial chambers. It seems that in the context of mitral valve disease increasing size of left atria certainly does not promote embolism and may indeed militate against it, for, as suggested in the $C$. introduction, platelet deposition is less rather than $\underset{\vec{F}}{\vec{*}}$ more likely in such chambers. Atrial fibrillation of $\stackrel{\text { f }}{\rightarrow}$ course is associated causally or sequentially with atrial $\frac{\text { 을 }}{2}$ enlargement, but it could be that it promotes embol- $\frac{O}{\bar{c}}$. ism neither by this association nor by the factor of $\frac{\overrightarrow{ }}{\vec{\alpha}}$ stasis, but because the intense and unceasing activity $\Omega$ of the muscle in the atrial wall has an adverse effect on $\%$ secretion of prostacyclin. This possibility merits $\vec{O}$ investigation.

The patients in subgroup 1B were, however, dis- $\vec{\omega}$ tinguished by their significantly greater age $(60: 47) \stackrel{\circ}{\partial}$ than those in group $1 \mathrm{~A}$ at the time of their first? embolism, again agreeing with Casella et al. 5 that $\overrightarrow{0}$ increased age is associated with increased risk of ? embolism. How this factor of age operates is not clear. $\vec{\omega}$ These same authors showed it was not related to car- 0 diac output. It is also not related to the age at onset of ${ }^{\supset}$ atrial fibrillation, because in this study this age was $\vec{T}$ almost identical in groups 2, 1A, and 1B. Possibly $\Phi$ shortened platelet survival after the age of 60 consistent with the finding of Dewar et al. ${ }^{22}$ of higher beta thromboglobulin values after this age could be the $\vec{\theta}$ important factor.

Although the levels of fibrinolytic activity, as measured by the euglobulin lysis time in the groups and응 subgroups, did not differ significantly, the mean values found especially in the women were all lower thano the mean value of 54 units found in the normal sub-\% jects previously measured by the method used in this@ study ${ }^{14}$ and the figure of 47.5 units of Chakrabarti $e t_{\vec{O}}^{\vec{B}}$ al. ${ }^{23}$ Previous experience with chronic rheumatic 3 heart disease patients and others ${ }^{13}$ suggests that in the cases in this study fibrinolytic activity in the left heart? including the atrium would be lower still. The estima-tions did not help to distinguish those liable to embol- $-\stackrel{\oplus}{2}$ ism from the rest, but the low values may nevertheless:be a factor in the generation of intra-atrial thrombi The very modest changes when patients were nonfasting and unrested suggest that the values remain 3 low for most of the day. Whether the low oxygeno saturation in the mixed venous blood which is so characteristic of mitral valve disease patients is the explanation of these low values is not certain. Goodhart and Dewar ${ }^{24}$ found no relation, but Per- $\infty$ lewitz and Markwartdt ${ }^{25}$ did find in endothelial cul- $N$ ture that a low oxygen content in the nutritionalu medium sharply diminished generation of fibrinolytic activator.

Although the values of beta thromboglobulin in the two groups and subgroups have not differed? significantly, they were all considerably higher than the normal values found in patients of the same age groups from blood taken and measured by Dewar exp $a l .{ }^{22}$ and also by others. ${ }^{26} 27$ They correlate well with the observations by Toy et al. ${ }^{9}$ of increased platele 
stickiness and by Steele et al. ${ }^{8}$ of shortened platelet survival in patients with mitral valve disease. Conard et al. ${ }^{28}$ in a small group of similar cases also found high mean values and Heilmann et al. ${ }^{29}$ in a much larger group considerably higher mean values of beta thromboglobulin. Our hope that the protein might be an easy and reliable marker of those patients with mitral valve disease and/or atrial fibrillation who ran serious risk of embolism and required preventive treatment against it or of those patients, especially those with recurrent embolism despite anticoagulants who needed antiplatelet drugs instead or as well, has not been fulfilled. Nor has the estimation correlated in any way with the type of mitral valve disease encountered.

So far as smoking is concerned the investigation has shed no light upon its importance or otherwise. Cigarette smoking has little effect on fibrinolytic activity ${ }^{14} 15$ but it does diminish the amount of circulating prostacyclin and production of it by vessel walls. ${ }^{29} \mathrm{It}$ also shortens platelet survival ${ }^{30}$ and Duncan et al. ${ }^{31}$ did find significantly higher blood levels of beta thromboglobulin among the non-smokers. In the present study, however, the higher values among current smokers as compared with non-smokers did not attain statistical significance, nor was there a greater prevalence of smoking in the high risk group $1 \mathrm{~B}$, in the enlarged embolic group at the time of their embolism, nor in the original embolic group at the crucial time of the onset of their atrial fibrillation as compared with the non-embolic group at the same time. The substantial reduction in the prevalence of the habit in both groups over the years of observation doubtless reflects the combined effect of medical advice and the changing pattern in the community.

The contraceptive pill was used so infrequently by both groups of patients in this study that in relation to embolism it had virtually no importance whatever.

The finding that a significantly larger proportion of emboli occurred between 0801 and 1600 hours than at other times is interesting, but one can only speculate upon reasons for it. The mechanical effect of nonrecumbency, changes in cardiac output-little though these are in most mitral valve cases-alterations in platelet aggregability, and adhesiveness in response to greater catecholamine secretion or changes in prostacyclin production can all be considered. The timing of the normal diurnal variation and the normal rise with exercise, even though it has proved to be very small, would both render changes in fibrinolytic activity an improbable factor.

We are much indebted to Dr Joan Dawes, MRC Immunoassay Team, Edinburgh for the estimations of beta thromboglobulin, to Dr P Smith, Royal Victoria Infirmary, Newcastle, for other biochemical tests, to colleagues in the Cardiovascular Department, Freeman Hospital, Newcastle, for permission to approach some of their patients, and finally to the patients themselves for their co-operation in the study.

\section{References}

1 Daley R, Mattingly TW, Holt CL, Bland EF, White PD. Systemic arterial embolism in rheumatic heart disease. Am Heart $\mathcal{F}$ 1951; 42: 566-81.

2 Wood JC, Conn HL Jr. Prevention of systemic arterial embolism in chronic rheumatic heart disease by means of protracted anticoagulant therapy. Circulation 1954; 10: 517-23.

3 Szekely P. Systemic embolism and anticoagulant prophylaxis in rheumatic heart disease. Br Med F 1964; i: 1209-12.

4 Fleming HA, Bailey SM. Mitral valve disease, systemic embolism and anticoagulants. Postgrad Med F 1971; 47: 599-604.

5 Casella L, Abelmann WH, Ellis LB. Patients with mitral stenosis and systemic emboli: hemodynamic and clinical observations. Arch Intern Med 1964; 114: 773-81.

6 Coulshed N, Epstein EJ, McKendrick CS, Galloway RW, Walker E. Systemic embolism in mitral valve disease. Br Heart f 1970; 32: 26-34.

7 Aberg $\mathrm{H}$. Atrial fibrillation. 1. A study of atrial thrombosis and systemic embolism in a necropsy material. Acta Med Scand 1969; 185: 373-9.

8 Steele PP, Weily HS, Davies H, Genton E. Platelet survival in patients with rheumatic heart disease. $N$ Engl f Med 1974; 290: 537-9.

9 Toy JL, Lederer DA, Tulpule AT, Tandon AP, Taylor $\mathrm{SH}, \mathrm{McNicol}$ GP. Coagulation studies in rheumatic heart disease. Br Hean f 1980; 43: 301-5.

10 Rozenberg MC, Dintenfass L. Thrombus formation in vitro: a rheological and morphological study. Aust $\mathcal{f}$ Exp Biol 1964; 42: 109-15.

11 Lackner H, Merskey C. Variation in fibrinolytic activity after acute myocardial infarction and after the administration of oral anticoagulant drugs and intravenous heparin. $\operatorname{Br}$ f Haematol 1960; 6: 402-13.

12 Nilsson IM, Pandolfi M. Venous cuff test. In: Davidson JF, Samama MM, Desnoyers PC, eds. Progress in chemical fibrinolysis and thrombolysis. vol. 2. New York: Raven Press, 1976: 3-6.

13 Menon IS, Weightman, D, Dewar HA. The role of the lung in blood fibrinolysis. Clin Sci 1969; 36: 427-33.

14 Menon IS. Fibrinolytic activity in the blood of 99 healthy young British, African and Asian subjects. Lab Pract 1968; 17: 194-5.

15 Janzon L, Nilsson IM. Smoking and fibrinolysis. Circulation 1975; 51: 1120-3.

16 Ellis LB, Harken DE. Arterial embolization in relation to mitral valvuloplasty. Am Heart $\mathcal{Y}$ 1961; 62: 611-20.

17 Graham GK, Taylor JA, Ellis LB, Greenberg DJ, Robbins SL. Studies in mitral stenosis. II. A correlation of post-mortem findings with the clinical course of the disease in 101 cases. Arch Intern Med 1951; 88: 532-47. 
18 Bolton AE, Ludlam CA, Moore S, Pepper DS, Cash JD. Three approaches to the radioimmunoassay of $\beta$-thromboglobulin. $\mathrm{Br} \mathcal{F}$ Haematol 1976; 33: 233-8.

19 Von Kaulla KN. Chemistry of thrombolysis: human fibrinolytic enzymes. Springfield, Illinois: Charles C Thomas, 1963: 79.

20 Menon IS, Muscat-Baron J, Dewar HA. Comparison of fibrinolytic activity in hepatic and systemic venous blood. F Atheroscler Res 1968; 8: 547-54.

21 Somerville W, Chambers RJ. Systemic embolism in mitral stenosis: relation to the size of the left atrial appendix. Br Med F 1964; ii: 1167-9.

22 Dewar HA, Marshall T, Weightman D, Prakash V, Boon PJ. $\beta$-thromboglobulin in antecubital vein blood-the influence of age, sex and blood group. Thromb Haemostas 1979; 42: 1159-63.

23 Chakrabarti R, Meade TW, North WRS, Stirling Y. Fibrinolytic activity in an industrial population. In: Davidson JF, Rowan RM, Samama MM, Desnoyers PC, eds. Progress in chemical fibrinolysis and thrombolysis. vol. 3. New York: Raven Press, 1978; 115-9.

24 Goodhart JM, Dewar HA. Fibrinolysis in pulmonary hypertension. F Atheroscler Res 1967; 7: 43-51.

25 Perlewitz J, Markwardt F. Studies on production and release of plasminogen activator in cell cultures. In: Davidson JF, et al. eds. Progress in chemical fibrinolysis and thrombolysis. vol. IV. Edinburgh: ChurchillLivingstone, 1979; 395-8.
26 Dawes J, Smith RC, Pepper DS. The release, distribu- $C$ tion, and clearance of human $\beta$-thromboglobulin and platelet factor 4. Thromb Res 1978; 12: 851-61.

27 Zahavi J, Cella G, Dubiel M, Kakkar VV. The variability of plasma $\beta$-thromboglobulin in healthy individuals. Thromb Haemostas 1978; 40: 565-7.

28 Conard J, Terrier E, Baillet $M$, et al. Variations de la $\beta$-thromboglobuline au cours de la contraception orale, de valvulopathies appareillees ou non, et de l'embolie pulmonaire. Nouv Presse Med 1981; 10: 1327-9

29 Heilmann E, Bender F, Breuer HWM, Klinke F. BetaThromboglobulin im Plasma bei Herzklappenfehlern und nach Herzklappenersatz. Z Kardiol 1980; 69: 815-7.

30 Fuster V, Chesebro JH, Byrne JM. Platelet survival and the development of coronary disease in the young: the effect of cigarette smoking, family history and medical therapy (abstract). Thromos Haemostas 1979; 42: 356.

31 Duncan A, DePratti VJ, George RR. Elevated $\underset{\omega}{\omega}$ $\beta$-thromboglobulin levels associated with smoking and oral contraceptive agents in normal healthy women. Thromb Res 1981; 21: 425-30.

Requests for reprints to Dr H A Dewar, The Royal Victoria Infirmary, Queen Victoria Road, Newcastle upon Tyne NE1 4LP. 